\title{
Michela Landi, Per un'organologia poetica. Gli strumenti musicali nella poesia francese romantica e simbolista
}

\section{Silvia Rovera}

\section{(2) OpenEdition \\ 1 Journals}

\section{Edizione digitale}

URL: http://journals.openedition.org/studifrancesi/8016

DOI: $10.4000 /$ studifrancesi.8016

ISSN: 2421-5856

\section{Editore}

Rosenberg \& Sellier

\section{Edizione cartacea}

Data di pubblicazione: 1 juillet 2009

Paginazione: 425-426

ISSN: 0039-2944

\section{Notizia bibliografica digitale}

Silvia Rovera, «Michela Landi, Per un'organologia poetica. Gli strumenti musicali nella poesia francese romantica e simbolista», Studi Francesi [Online], 158 (LIII | II) | 2009, online dal 30 novembre 2015, consultato il 07 janvier 2021. URL: http://journals.openedition.org/studifrancesi/8016 ; DOI: https:// doi.org/ERREUR PDO dans /localdata/www-bin/Core/Core/Db/Db.class.php L.34 : SQLSTATE[HY000] [2006] MySQL server has gone away

Questo documento è stato generato automaticamente il 7 janvier 2021.

\section{(†)

Studi Francesi è distribuita con Licenza Creative Commons Attribuzione - Non commerciale - Non opere derivate 4.0 Internazionale. 


\title{
Michela Landi, Per un'organologia poetica. Gli strumenti musicali nella poesia francese romantica e simbolista
}

\author{
Silvia Rovera
}

\section{NOTIZIA}

MICHELA LANDI, Per un'organologia poetica. Gli strumenti musicali nella poesia francese romantica e simbolista, Tomo I, Aerofoni e cordofoni, Firenze, Leo S. Olschki Editore, 2008, pp. 489.

1 Il volume di Michela Landi traccia il percorso degli strumenti musicali nella poesia francese dell'Ottocento, partendo dal simbolismo degli aerofoni e dei cordofoni, associati ora alle idee di cui sono un'amplificazione (com'è il caso delle corde, che lasciano libero corso al fluire della parola), ora a un corpo vile e sedizioso (come i fiati che impediscono la voce). A questa breve premessa, l'autrice fa seguire l'analisi dei vari strumenti, in primis quella del corno.

2 Strumento venatorio, usato per catturare l'alterità dell'animale ucciso, esso si trasforma poco a poco nell'attributo dell'eroe culturale. Questo è il caso dell'olifante di Roland, il cui suono, preludio della morte, diventa l'emblema di una soggettività solitaria, la stessa che compare a fine ' 800 , nella Parigi straniante e desertica di Baudelaire. La stessa nostalgia per un "Paradis enfantin" o "latin" si ritrova in Verlaine, che sente risuonare attraverso il corno il nome perduto di Mathilde, la cui presenza è tale solo grazie al riverbero acustico.

Di significato contrario, vale a dire euforico, è la tromba, legata, per le virtù apotropaiche del suo materiale, alla rappresentazione dell'oro e della luce. Così, in Baudelaire, è associata al metallo monetato (simbolo della "civilisation") o alchemico (l'“or sonore" del poeta), mentre in Mallarmé (nella rivisitazione della lotta di Giacobbe con l'angelo) è unita all'archetipo del sole come cacciatore. Come attributo iconico della 
rivelazione divina compare in Hugo, attraverso le trombe di Gerico, e in Baudelaire, che, ne Les tentations, la trasforma in un "instrumentum damnationis", legandola a una divinità che ha spesso tratti caniformi (Le voyage) o un corpo vuoto e perennemente affamato (Madrigal triste, Voyage).

Ben diverse sono le caratteristiche del flauto. Associato alle cerimonie falliche, ha una doppia funzione: attiva (fecondativa) e punitiva (nell'analogia morfologica con il "flagellum"). Nell'Ottocento è l'attributo sovversivo del suonatore ambulante, eroe portatore d'istanze sciamanico-profetiche, ma al contempo denigrato. È quello che trapela dal poema di Vigny, La flute, mentre in Verlaine risponde al progetto poetico di una dissoluzione dell'identità, trovandosi spesso in diadi con "volute", termine che si lega alla danza e alla guerra d'amore di stampo baudelairiano. Mallarmé, invece, lo considera "instrument direct de l'idée" e lo riconduce ancora una volta all'ambiguità del femminino, materno e cullante, ma anche aggressivo e seduttivo, come si nota in Sainte, dove diventa l'emblema della sensualità di cui si sbarazza la donna. Al contrario, nell'Après-midi d'un faune, l'abolizione dello strumento diventa il paradigma della poesia moderna, che ha spezzato il suo legame con l'oggetto, cioè con la musica.

Più titolato appare l'organo, definito "rex instrumentorum" e rivestito, fin dal Medioevo, di una funzione sacra tanto da essere frequentemente assimilato alla cattedrale o al corpo umano. Dopo un breve periodo d'oscurantismo, riacquista fulgore in epoca romantica, grazie alla sua versione elettro-pneumatica che smaschera il rito come simulacro della presenza divina e lo avvicina alla "jonglerie". Per tale motivo, in Wagner corrisponde alla degenerazione della musica stessa, poiché la sua varietà ritmica è slegata dalla voce umana.

6 Di carattere simile è l'organetto di Barberia, che, a metà tra gli strumenti aerofoni e quelli meccanici, si fa il simbolo della reificazione dell'uomo moderno e del suo asservimento alla logica della produzione. Nel xIX secolo, infatti, è lo strumento ad avere la ridondanza psicologica e identitaria dell'esecutore, assumendone la colpa morale e divenendo "instrument des tristes", come dirà Mallarmé. Pertanto le sue peculiarità fono-simboliche ora sono rielaborate in lamento, ora hanno le connotazioni sacrificali della sega (basti ricordare Baudelaire dei Poèmes à faire e dello Spleen de Paris). Altre volte il suo suono serve come "medicina doloris", alleggerendo la "lourdeur" fisica e metafisica di un lavoro inteso come coazione (Laforgue e Verlaine).

7 Legati ad altre istanze sono i cordofoni, simboli della misura e dell'ordine e, dunque, del mesocosmo. Le loro sette corde rimandano ai pilastri del tempio della saggezza, mentre la tensione che richiedono all'uomo è edificatoria, poiché li trasforma nel presupposto ideomimetico della comprensione e della concentrazione di un'energia materiale e spirituale.

8 Tra questi occupa un posto di primo piano il violino. In Francia è il protagonista incontrastato delle feste e dei balli, e, di conseguenza, della "concordia universalis", in quanto suonato "a plusiori". Tuttavia il suo timbro marcato, imitatore della voce femminile, porta molti scrittori a definirlo empio, sia per la sua tracotanza, sia perché i suoi acuti scatenano sofferenza nei soggetti nervosi. L'analogia con la donna si riscontra anche nella cassa di risonanza a mandorla, simbolo del ventre materno o dell'anima di Eco, come ricorda Baudelaire in Le voyage à Cythère. L'immolazione della ninfa assimilata al poeta è altresì presente nella Chanson d'automne verlainiana, in cui il singulto ritmico del violino corrisponde all'istanza ecforica e distruttiva del ballo. 
9 Rappresentativi della poesia come canto sono invece la lira e l'arpa. La lira, la cui origine sacrificale è stata riscoperta dall'ottocento romantico e simbolista, ispira un gran numero di poeti. Se Lamartine la vede santificata dal suo sacrificio terreno, Hugo la fa assurgere a paradigma dei sette peccati capitali e la trasforma in uno strumento di tortura. La stessa sofferenza sarà rivisitata in ottica impressionista da Verlaine, che la ripropone nella diade "lyre/délire", e da Mallarmé, che, pur preferendole la cetra, la vede come "medium" della "disparition vibratoire" dell'oggetto. L'arpa, di moda nello stesso periodo, testimonia della decadenza del modello greco-latino in favore di quello cristiano-giudaico. Il suo carattere sacro è ben evidenziato, oltre che dalla forma ad ala, anche dal numero di corde: dieci come il Decalogo. Baudelaire la riprende proprio in quest'accezione, unendola alla figura del cigno e a quella del saltimbanco.

10 L'elenco potrebbe continuare ancora per un po': l'opera della Landi è piena di rimandi e citazioni che spesso s'intrecciano tra di loro, molte volte riprendendosi e ripetendosi, perché lo scambio di percezioni tra la poesia e la musica è fatta da autori che seguono o contestano i loro predecessori. Crediamo che il secondo volume, dedicato ai membranofoni e agli idiofoni, riesca a illuminarci ulteriormente su un'epoca che ha cercato di riunire stimoli sensoriali sempre più divisi dal settorialismo capitalista. 